\title{
Unique Case of Isolated Jejunal Angiodysplasia Refractory to Coil \\ Embolization Managed by Laparoscopic Resection
}

\author{
Jyotsna Misra ${ }^{1 *}$, Subham Bera ${ }^{1}$, Shuvro H Roy Chowdhury ${ }^{2}$ and \\ Shubhayu Banerjee ${ }^{3}$ \\ ${ }^{1}$ DNB Resident, Department of General Surgery, Rabindranath Tagore International \\ Institute of Cardiac Sciences, Kolkata, West Bengal, India \\ ${ }^{2}$ Consultant Intervention Radiology. NH Rabindranath Tagore International Institute \\ of Cardiac Sciences. Kolkata. West Bengal India \\ ${ }^{3}$ Consultant Laparoscopic and Gastrointestinal Surgeon. Rabindranath Tagore \\ International Institute of Cardiac Sciences. Kolkata. West Bengal India \\ *Corresponding Author: Jyotsna Misra, DNB Resident, Department of General \\ Surgery, Rabindranath Tagore International Institute of Cardiac Sciences, Kolkata, \\ West Bengal, India.
}

Received: March 16, 2020

Published: March 30, 2020

(C) All rights are reserved by Jyotsna Misra., et al.

\section{Abstract}

Angiodysplasia is one of the commonest vascular lesion leading to gastrointestinal bleed accounting for approximately $6.0 \%$ of lower gastrointestinal (GI) bleeding and 1.2 - 8.0\% upper GI bleed. In elderly patients small bowel angiodysplasia is responsible for $40 \%$ of obscure bleed while in $10 \%$ of young adults small bowel angiodysplasia is the single most common cause of obscure GI bleed [1]. Jejunum is the most common site of intestinal vascular ectasia followed by ileum and duodenum [2]. By far degenerative changes are considered as the most common cause of upper intestinal angiodysplasia. Angiodysplasia has a varied clinical presentation, from a spectrum of occult bleeding to acute massive hemorrhage. It can also be diagnosed incidentally. CT angiography and endoscopy are the useful diagnostic tools. Therapeutic strategies include or angiographic embolization, endoscopic obliteration of bleeding angiodysplastic lesion and Surgery [3].

Keywords: Angiodysplasia; CT Angiography; Laparoscopic Resection

\section{Introduction}

Goldabine first described angiodysplasia as an ectasia of the submucosal vessels of the bowel [4]. Further it has been seen to be an abnormal ectatic capillary in the mucosal and submucosal layer of GI tract consisting endothelium with minimal or absent smooth muscle which can readily rupture and cause bleeding [5]. Various management options for angiodysplasias includes endoscopic obliteration or angiographic embolization by intra-arterial vasopressin injection. Selective gel foam embolisation, endoscopic electrocoagulation and injection of sclerosants are also other modalities used in treatment of angiodysplasia [6].

Microcoil embolization is a safe an efficacious method in the management of Acute GI hemorrhage. Operative intervention has been indicated for refractory bleeding or lesions in sites unaccessible to endoscopic interventions. Coil embolization prior to surgery is also useful for the localization of the lesion as the coil is palpable intraoperatively in open surgery and detectable under X-ray fluoroscopy in both open and laparoscopic surgery. The challenge is more in laparoscopic surgery due to limited viewing area on the screen, handling bowel loops and absence of tactile feedback of Coil as compared to open surgery. We are reporting a case of 57 year old lady with an isolated jejunal angiodysplasia refractory to coil embolization and managed by laparoscopic resection.

\section{Case Report}

A 57 years old lady presented with dyspnoea on exertion, generalised weakness and repeated episodes of Malena. On further questioning she dated her malena for 3 - 4 years with a background of hypertension and diabetes. Her initial diagnosis was Acute Coronary Syndrome, Congestive Heart failure and Anemia (Hb\% 7.7 $\mathrm{gm} / \mathrm{dl}$ ). The Acute coronary syndromes was treated first and underlying anemia was corrected ( 2 units of packed cell transfusion). Upper GI endoscopy revealed antral erythema and colonoscopy confirmed rectal and colonic polyps, histopathology confirmed it as Tubular adenoma but all of the above investigations could not delineate the source of bleeding.

She was readmitted after 1 month with recurrent Malena and Anemia (Hb\% 7.8 gm/dl). Antiplatelet medications were kept on hold and she was further evaluated. Capsule Endoscopy failed to identify any source of Intestinal bleed. CT angiography confirmed angiodysplasia of second Jejunal branch of SMA with active extravasation (Figure 1 and 2). Coil embolization was performed (Figure 3 and 4), she remained stable with no further decrease in Hemoglobin and discharged without any complications. 


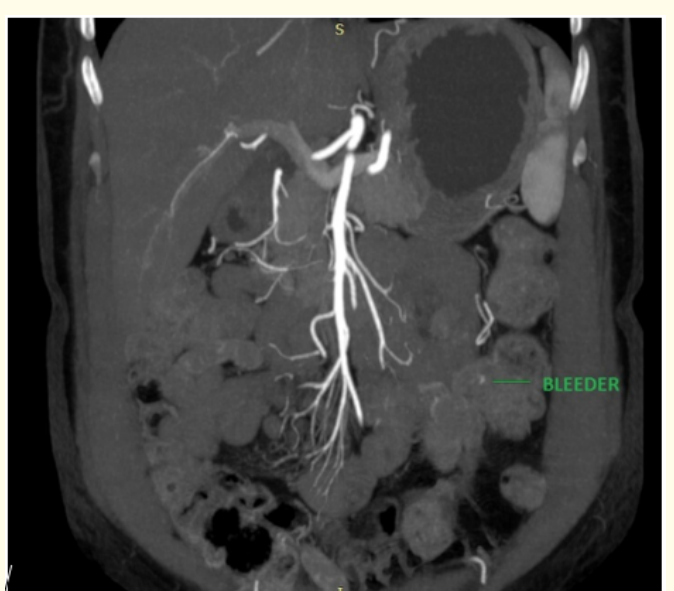

Figure 1: CT Angiogram maximal intensity projection image showing active bleed from second jejunal branch.

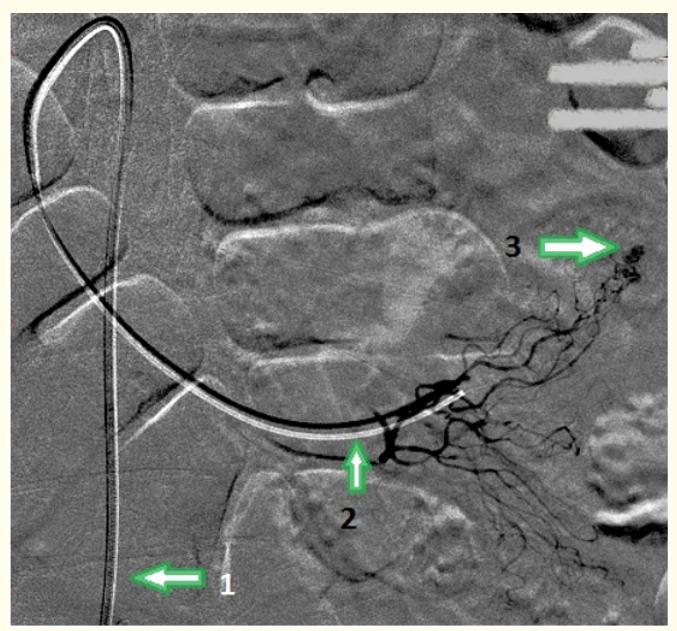

Figure 2: Angiogram after super selective cannulation of jejunal branch of superior mesenteric artery with a micro catheter showing active arterial blush (Arrow 3). Arrow 1: Parent catheter, Arrow 2: Micro catheter.

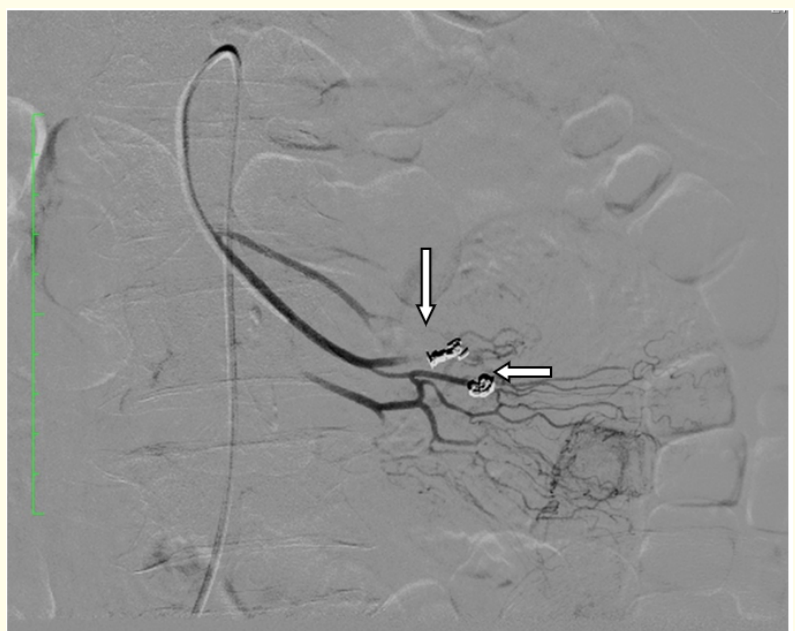

Figure 3: Angiogram after super selective coiling arrows demonstrating front door and back door coils.

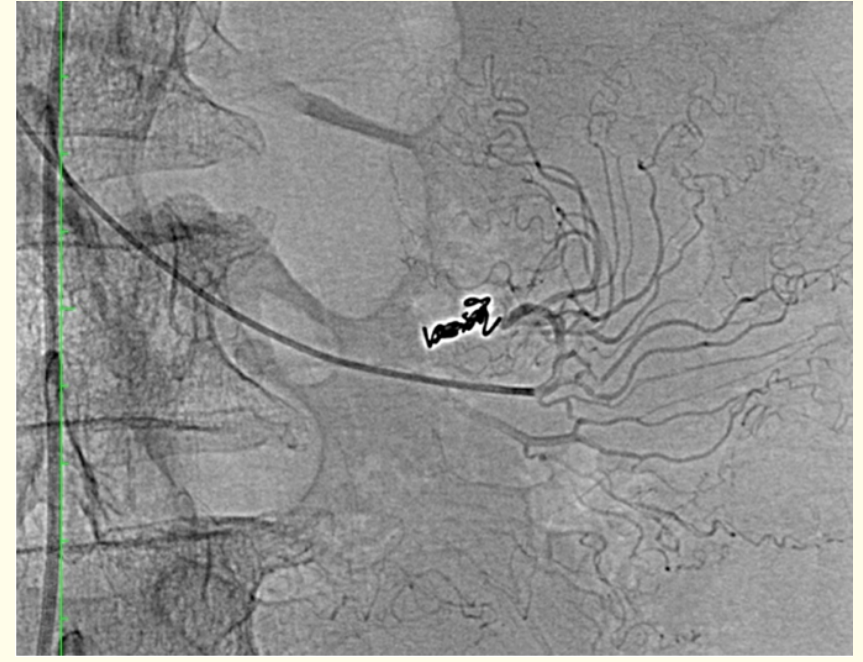

Figure 4: Post coil embolization angiogram demonstrating no active arterial blush from second jejunal branch of SMA.

Within 1 month of embolization she re-presented with malena. On admission her hemoglobin was $5 \mathrm{gm} / \mathrm{dl}$. Repeat CT Angiography confirmed a small recurrence with active bleeding from a side branch of previously embolized vessel. However, because of the risk of another rebleed, and possibility of necrosis of the coil-embolized jejunum, we recommended resection of the affected jejunual loop to be the optimal treatment. Diagnostic laparoscopy was performed and with the aid of fluoroscopy (Figure 5), coil was localized and resection of $10 \mathrm{~cm}$ of Jejunal segment $(5 \mathrm{~cm}$ proximal and $5 \mathrm{~cm}$ distal to the coil) and a functional end to end anastomosis was done. Postoperative period remained uneventful and she was discharged within 2 weeks. She did not have any further episode of malena and had normal hematocrits during her 3 months of Follow-up.

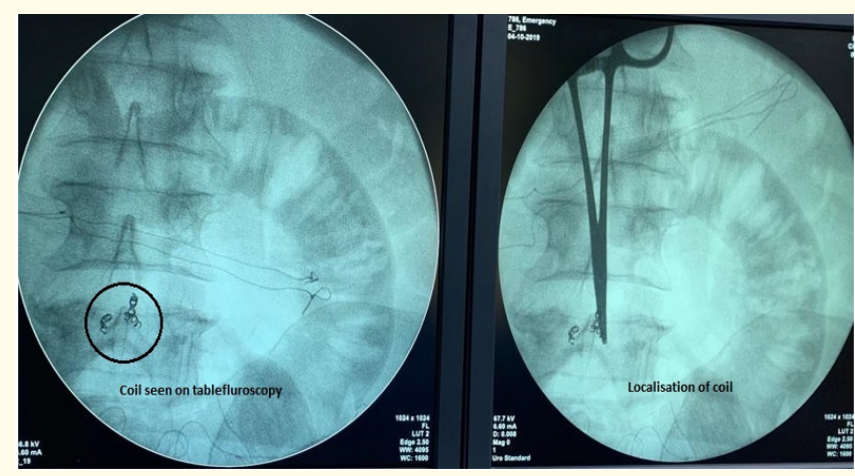

Figure 5: Intra-operative coil localization under X-ray fluoroscopy.

\section{Discussion}

Obscure gastrointestinal bleed is defined as any bleeding in the gastrointestinal tract from oesophagus to the rectum. Various causes includes oesophageal tear or inflammation in the oesophagus, peptic ulcer or perforation, diverticulosis and diverticulitis, inflammatory bowel disease, colonic polyps, vascular ectasias/ malformations, ca colon, stomach or esophagus and hemorrhoids. 


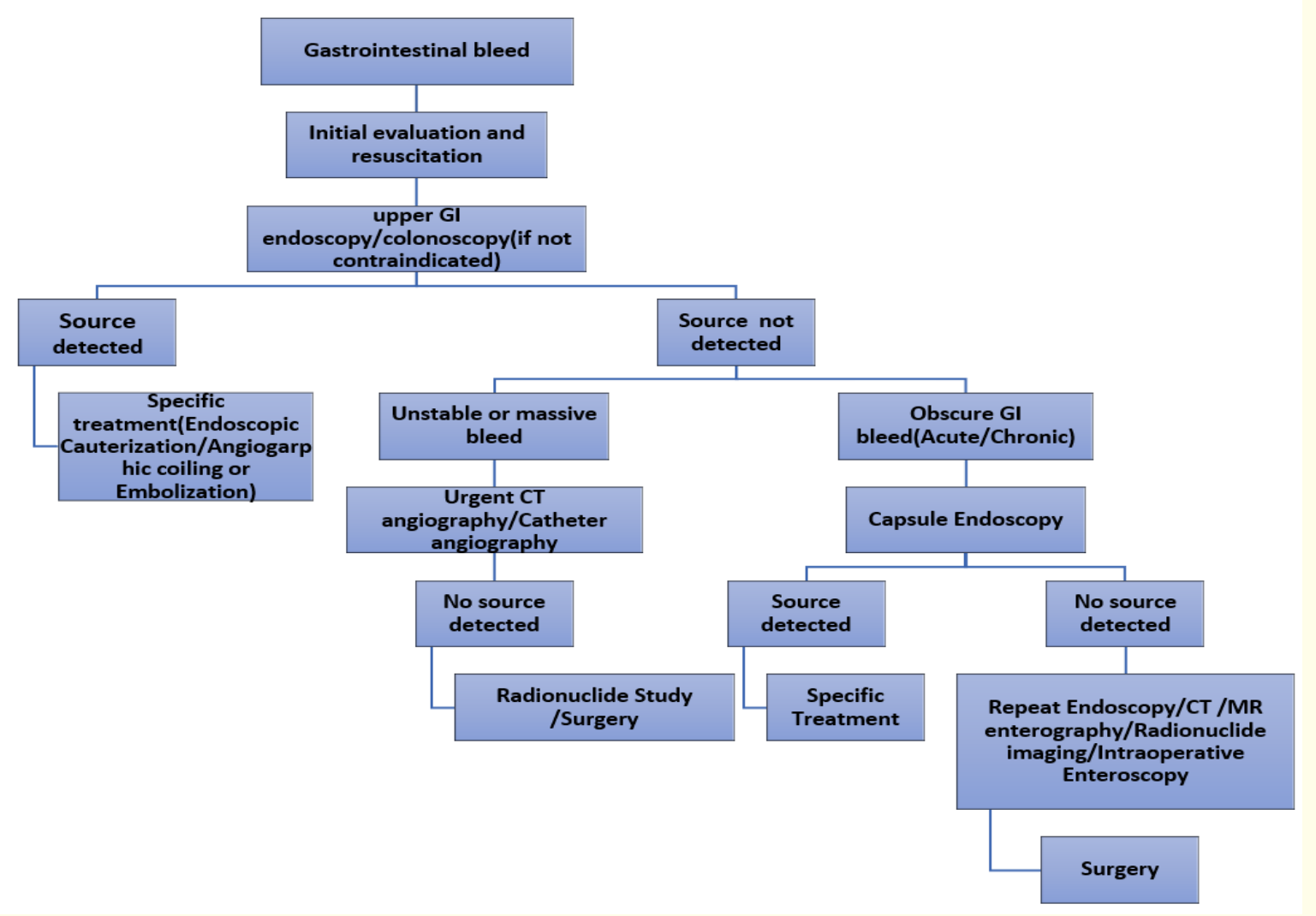

Figure

Aberrant blood vessels are probably more common in GI tract than anywhere else in the body. They can be present since birth or develop as part of inherited syndromes, but the vast majority are acquired later in life with advancing age. Angiodysplasia of gastrointestinal tract is a pathologically expansile blood capillary most commonly found in colon. In patients older than 50 year of age small bowel angiodysplasia is the most common cause of obscure GI bleeding. Exact pathophysiology of angiodysplasia still remains unclear, most accepted hypothesis is that chronic obstruction at the level of the muscular propria and submucosa of these capillaries may predispose to age-related angiodysplastic lesion. Arteriovenous collaterals develops as a result of capillary and precapillary congestion [7]. 30\% - 40\% of angiodysplasia is of indeterminate cause [8]. Mostly it is diagnosed incidentally but it could also present as recurrent malena, or with subtle symptoms of anemia. There are various therapeutic options such as endoscopy, vasopressin infusion and surgery. It is difficult to prove that diagnostic endoscopy improves the outcome, but it is clearly important to define a precise diagnosis in order to plan treatment, hence it is often considered as the first method of choice (Failure rate up to 32\%) [9]. Surgery is the definitive treatment option, But as most of the patients with angiodysplasia are elderly, with numerous co-morbidities, such as cardiovascular disease, chronic kidney disease, and on anticoagulants and antiplatelet agents have relatively poor tolerance for surgery and the mortality rate can reach $9 \%$ to $47 \%$ [10].
In 1974, Bookstein., et al. first described treatment of angiodysplasia with intra-arterial embolization [11]. Angiographic endovascular embolization has emerged as one of the method in management of lower GI bleed (sensitivity 58\% to 86\%) [12]. Extravasation of the contrast medium representing active bleeding can be demonstrated in only $6 \%$ to $20 \%$ of patients [13]. During the arterial phase of angiography angiodysplasia lesion appears as opacified, dilated, tortuous, and slowly emptying intramural veins [14]. Embolic agents such as polyvinyl alcohol (PVA), Gelfoam, coils, autologous coagulum, and n-butyl cyanoacrylate (NBCA) are commonly used [15]. NBCA glue is highly effective as it can travel in as liquid embolic right up to the nidus of the abnormality, at the vasa recta level, thereby reducing the risk of rebleed from the collateral flow. However, its use is riskier as it can cause non target embolisation. But coils are mostly preferred as they are well visualized during direct fluoroscopy, have greater accuracy and precision of occlusion of the bleeding vessel, they avoid the reflux of particles, and also fulfill the objective of reducing the perfusion pressure while allowing collateral flow to prevent the infarction. It is a powerful method for treating acute GI bleed occurring due to Arterio-Venous malformations. Successful embolization occurs in $80 \%$ to $100 \%$ cases but chances of early rebleed are reported between $10 \%$ to $30 \%$. Probable cause of rebleed is either due to recanalization of the previously embolized site or new sources of bleeding in the GI tract [16]. Most dreaded complication of embolization, which is usually correlated with the number of embolized 
straight arteries is intestinal infarction (22\%). This can be prevented with superselective coil embolization of abnormal vessels [17]. Surgical Interventions are needed when all other above mentioned methods fails but localization of the angiodysplastic lesion during the surgery is difficult. Recently, Ono et al. reported that while performing the open surgery intraoperative indocyanine green dye injection and examination with a fluorescent scope could localize jejunal Arterio-Venous malformations [18]. This procedure is difficult during laparoscopic surgery due to limited view area on a screen. Another advantage of coil embolization is that it could also help in localization as the coil is palpable intraoperatively during an open surgery and are easily detectable under X-ray fluoroscopy in both laparoscopic and open surgery [19].

In this case, Superselective coil embolization of second jejunal branch was performed, patient did not show any acute complication after embolization. Unfortunately, rebleed occurred after 1 month. As our patient has history of IHD and was on antiplatelet medications, risks of rebleed and ischemia of jejunal segment associated with recoiling could have been deadly complications. Hence decision of surgical intervention is taken and she was successfully treated.

\section{Conclusion}

Superselective coil embolization is safe and effective method in the management of gastrointestinal tract bleed due to angiodysplasia and it is considered as a definitive treatment for stable patients without the need for further surgical resection. But the rate of recurrent bleeding after a successful coil embolization is higher in elderly patients, known Cardiac diseases and on antiplatelet therapy. In those cases, re-embolization may frequently lead to another rebleed or Ischemia of bowel segment. Therefore, surgery is the definitive option in this subset of patients. We report a case of jejunal Angiodysplasia initially managed with superselective coil embolization followed by laparoscopic resection of affected jejunal segment that was guided fluoroscopically. It highlights the effectiveness of the combination of superselective coil embolization and laparoscopic resection for patients with repetitive bleeding from a jejunal angiodysplasia.

\section{Bibliography}

1. Crabtree K. "Angiodysplasia of the Gastrointestinal Tract". Radiology 194.1 (1995): 96.

2. Sharma V., et al. "Gastrointestinal bleeding in the tropics: look for the hookworm”. Tropical Doctor 47.1 (2017): 48-51.

3. Chen H., et al. "Bleeding recurrence in patients with gastrointestinal vascular malformation after thalidomide". Medicine 95.33 (2016): e4606.

4. Pires D., et al. "Gastrointestinal angiodysplasia. Estrogenprogesterone therapy". Acta Medica Portuguesa 7.11 (1994): 631-633.

5. Höchter W., et al. "Angiodysplasia in the colon and rectum". Endoscopy 17.5 (1985): 182-185.

6. Xu M., et al. "Embolization of arterial gastrointestinal hemorrhage with Fuaile medical adhesive". Journal of the Chinese Medical Association 81.7 (2018): 636-642.
7. Triadafilopoulos G. "Management of lower gastrointestinal bleeding in older adults". Drugs and Aging 29.9 (2012): 707715.

8. Brown C., et al. "Somatostatin analogues in the treatment of recurrent bleeding from gastrointestinal vascular malformations: an overview and systematic review of prospective observational studies". Digestive Diseases and Sciences 55.8 (2010): 2129-2134.

9. Ali M., et al. "Treatment of nonvariceal gastrointestinal hemorrhage by transcatheter embolization". Radiology Research and Practice (2013): 604328.

10. Suh SW., et al. "Small bowel ischemia after angiographic embolization for angiodysplasia of lower gastrointestinal tract: the case for conservative management". Clinics and Research in Hepatology and Gastroenterology 35.12 (2011): 819-822.

11. Bookstein JJ., et al. "Transcatheter hemostasis of gastrointestinal bleeding using modified autogenous clot". Radiology 113.2 (1974): 277-285.

12. Hasanefendioglu Bayrak A., et al. "Occult bleeding of small bowel: endovascular embolization and literature review". Journal of Digestive Diseases 10.2 (2009): 152-156.

13. Richardson JD., et al. "Bleeding vascular malformations of the intestine”. Surgery 84.3 (1978): 430-436.

14. Nguyen N., et al. "Embolotherapy for small bowel angiodysplasia”. Gastrointestinal Endoscopy 58.5 (2003): 797-800.

15. Funaki B., et al. "Superselective microcoil embolization of colonic hemorrhage". American Journal of Roentgenology 177.4 (2001): 829-836.

16. Raphaeli T and Menon R. "Current treatment of lower gastrointestinal hemorrhage". Clinics in Colon and Rectal Surgery 25.4 (2012): 219-227.

17. Maleux G., et al. "Long-term outcome of transcatheter embolotherapy for acute lower gastrointestinal hemorrhage”. American Journal of Gastroenterology 104.8 (2009): 2042-2046.

18. Ono H., et al. "Intraoperative localization of arteriovenous malformation of a jejunum with combined use of angiographic methods and indocyanine green injection: Report of a new technique". International Journal of Surgery Case Reports 29 (2016): 137-140.

19. So M., et al. "Laparoscopic resection of idiopathic jejunal arteriovenous malformation after metallic coil embolization". Surgical Case Reports 4.1 (2018): 78.

\section{Assets from publication with us}

- Prompt Acknowledgement after receiving the article

- Thorough Double blinded peer review

- Rapid Publication

- Issue of Publication Certificate

- High visibility of your Published work

Website: www.actascientific.com

Submit Article: www.actascientific.com/submission.php Email us: editor@actascientific.com Contact us: +919182824667 